\title{
Development and preliminary evaluation of a short self-report measure of generalized pain hypersensitivity
}

This article was published in the following Dove Medical Press journal: Journal of Pain Research

\author{
Peter F van Bemmel' \\ Martijn AH Oude Voshaar ${ }^{2}$ \\ Peter $M$ ten Klooster ${ }^{2}$ \\ Harald E Vonkeman ${ }^{1,2}$ \\ Mart AFJ van de Laar ${ }^{1,2}$ \\ 'Department of Rheumatology, \\ Medisch Spectrum Twente, Enschede, \\ Netherlands; ${ }^{2}$ Department of \\ Psychology, Health, and Technology, \\ University of Twente, Enschede, \\ Netherlands
}

\begin{abstract}
Purpose: Generalized pain hypersensitivity is frequently observed in chronic pain conditions. Currently, identification is based on expert clinical opinion, and in very few cases combined with quantitative sensory testing. The objectives of this study were to develop and evaluate a short self-report measure of generalized pain hypersensitivity: a generalized pain questionnaire (GPQ). Methods: Items for the GPQ were developed based on a literature review, followed by an interview study with ten rheumatic patients with suspected pain hypersensitivity. We examined the psychometric properties of the preliminary items in a sample of 212 outpatients suffering from either fibromyalgia (FM; $n=98)$ or rheumatoid arthritis $(n=114)$. Additionally, self-reported data were gathered on sociodemographics, fibromyalgia-survey criteria, health status, and neuropathic-like pain features.
\end{abstract}

Results: Mokken-scale analyses demonstrated a unidimensional seven-item scale with strong homogeneity $(H=0.65)$ and high reliability $(\rho=0.90)$. Correlations between total GPQ scores and relevant external measures, such as the FM-survey criteria and neuropathic-like pain features, were consistent with a priori expectations, supporting its external construct validity. Furthermore, the GPQ had good accuracy in distinguishing between patients with FM (generally assumed to be the result of central nervous system hypersensitization) and patients with RA (assumed to result mostly in local nociceptive or inflammatory pain), with an area under the receiver-operating characteristic curve of 0.89 . A cutoff value $>10$ had the highest combination of sensitivity (82.7\%) and specificity (77.2\%).

Conclusion: The GPQ is psychometrically sound and appears promising for measuring the presence and severity of generalized pain hypersensitivity in chronic pain patients.

Keywords: central sensitization, chronic pain, nociceptive pain, questionnaires

\section{Introduction}

Chronic pain is a major health problem with an estimated prevalence of $17 \%-31 \%$ in developed countries. ${ }^{1-3}$ Traditionally, chronic pain has been classified into two broad categories: pain in response to peripheral tissue damage or inflammation, and pain associated with damage to or a lesion of the nervous system (neuropathic pain). ${ }^{4,5}$ Both are characterized by pain hypersensitivity at the injury site and in adjacent tissue as a manifestation of neuronal plasticity. ${ }^{6-8}$

Many patients with chronic pain, however, show symptoms of generalized pain hypersensitivity. Using quantitative sensory testing (QST), Schliessbach et $\mathrm{al}^{9}$ and Curatolo et $\mathrm{al}^{10}$ found that generalized pain hypersensitivity is common in patients with various chronic pain conditions. It is generally thought that generalized pain
Correspondence: Peter $\mathrm{M}$ ten Klooster Department of Psychology, Health, and Technology, University of Twente, PO Box 217, Enschede 7500 AE, Netherlands

Tel +3I 534896056

Email P.M.tenKlooster@utwente.nl 
hypersensitivity is a manifestation of central sensitization. ${ }^{11,12}$ Central sensitization is an increased responsiveness of nociceptive neurons in the central nervous system to their normal or subthreshold afferent input. ${ }^{6,13}$ It is characterized by enhanced spontaneous neuronal activity, enlarged receptive-field areas, and an increase in responses evoked by primary afferent fibers. ${ }^{14}$ Central sensitization is an adaptive physiological process that occurs in everybody, and normally it stops after termination of the nociceptive afferent input and returns to baseline. It is hypothesized that in chronic pain patients, this adaptive process persists, despite elimination of most nociceptive input. ${ }^{15}$ Additionally, endogenous descending pain facilitatory modulatory systems from the brain contribute to pain hypersensitivity. ${ }^{16}$ These mechanisms are some of the most important currently known; however, knowledge about pain mechanisms is constantly evolving. Generalized pain hypersensitivity typically manifests as allodynia, (secondary) hyperalgesia, and aftersensations, ${ }^{11,12}$ and is a characteristic feature of fibromyalgia (FM). ${ }^{12,17-23}$

Accurate pain measurement and classification of clinical pain phenotypes is essential for adequate pain treatment. In clinical practice, the recognition of generalized pain hypersensitivity is generally based on the clinician's evaluation of symptoms and medical history, occasionally combined with QST using thermal, electrical, and/or mechanical stimuli. ${ }^{22-25}$ QST methods, however, are complex and time-consuming, and thus often impractical in daily clinical care or research settings. ${ }^{26}$ Consequently, a self-report measure of generalized pain hypersensitivity could provide a time-efficient method for the initial identification of patients with elevated pain sensitivity. Moreover, such a measure could be useful for evaluating efficacy of treatments specifically targeting generalized hypersensitivity to pain.

The objectives of this study were to develop and evaluate a short and easy-to-use self-report instrument for identifying the presence and intensity of generalized pain hypersensitivity. We subsequently explored the psychometric performance of this generalized pain questionnaire (GPQ) in patients with FM and rheumatoid arthritis (RA).

\section{Methods}

\section{Development of items}

An initial set of symptoms characteristic of or associated with generalized pain hypersensitivity was derived from a literature review and reviewed by the project team. A pragmatic interview study was then performed on patients with suspected pain hypersensitivity in September 2014. Patients were recruited from participants in a previous survey in which the PainDetect questionnaire (PDQ) was administered. ${ }^{27} \mathrm{We}$ expected that ten patients would be necessary to obtain appropriate patient descriptions and examples of these symptoms. Patients with high PDQ total scores and divergent scores on items suggestive of allodynia and hyperalgesia were contacted by phone to ask if they were interested in participating in an interview study about their pain symptoms.

After 23 patients had been contacted, 10 agreed to participate in the study, of which 7 were female. Interviews were held at the participants' homes. Using a semistructured interview scheme, participants were asked if they recognized the symptoms of pain hypersensitivity identified in the literature, and if so, to describe their personal experience with these symptoms in their own words.

As the aim of the interviews was to develop understandable items and recognizable examples of manifestations of symptoms identified in the literature, no formal inductive coding or thematic analyses of the data were undertaken. Instead, patients' comments, descriptions, and examples were categorized by symptoms. Based on this, preliminary items were developed by one of the authors (MOV), who also performed the interviews, and discussion with two of the other authors (PvB, PtK) for item clarity and relevance. This resulted in seven items covering typical symptoms of generalized pain hypersensitivity described in the patient's language and with common examples that patients mentioned during the interviews. To measure the severity of the symptoms, all items were provided with a 5-point Likert-type rating scale $(0=$ never, $1=$ hardly noticed, $2=$ moderately, $3=$ strongly, $4=$ very strongly), based on the response options of the PDQ. ${ }^{28}$ Like the PDQ, no recall period was added.

\section{Psychometric evaluation Study population and data collection}

The preliminary items were administered to two crosssectional samples of patients with clinically established FM or RA from the rheumatology outpatient department of the Medisch Spectrum Twente hospital in Enschede, the Netherlands. The FM patients were identified using the ICD10 code M79.7. In December 2016, all FM patients who had visited the hospital for consultation with a rheumatologist or rheumatology nurse between November 2015 and October 2016 were invited by postal mail to participate in this survey study. In total, 99 of 196 invited patients (51\%) returned the paper-and-pencil questionnaire. All FM patients received one reminder. RA patients completed the same questionnaires as the FM patients. Data for RA patients were collected within the Dutch Rheumatoid Arthritis Monitoring (DREAM) RA 
registry. The DREAM-RA is a quality registry in which clinical and patient-reported data from patients with a clinical diagnosis of RA are prospectively registered. Between January 2017 and March 2017, RA patients were invited to participate after logging in to their online patient portal. In total, $114 \mathrm{RA}$ patients completed the preliminary items.

According to the Dutch Medical Research Involving Human Subjects Act, the interview and survey study did not need formal approval of a medical ethical review board, as they were observational nonintervention studies, without a high burden to patients. Therefore, written informed consent was not obtained for the interview or survey study. However, in both studies, all patients were fully informed about the goal of the study and the voluntary nature of participation.

\section{Other measures}

Besides the seven preliminary generalized pain items, all patients were asked to complete several other standardized patient-reported outcome measures (PROMs). All PROMs were administered in Dutch, and (except for the FM-survey questionnaire), had previously been validated for use in Dutch patient populations. Additional self-reported information was obtained on sociodemographics, the use of conventional (eg, paracetamol, nonsteroidal anti-inflammatory drugs, or opioids) and neuropathic (central nervous acting drugs such as amitriptyline, nortriptyline, gabapentin) pain killers, and on disease duration.

The Short Form health survey (SF36 version 2) was used to measure health-related quality of life using 36 questions assessing eight aspects of health: physical functioning, role - physical, bodily pain, general health, vitality, social functioning, role - emotional, and mental health. ${ }^{29,30}$ These dimensions can be summarized into a physical component summary and a mental component summary. These summaries are standardized using normative data from the US population with a mean of 50 and a SD of 10 .

Physical disability was measured with the Health Assessment Questionnaire - disability index (HAQ-DI). ${ }^{31,32}$ The HAQ-DI consists of 20 questions divided over eight categories (dressing, arising, eating, walking, hygiene, reach, grip, and usual activities). The highest score in each of the categories are added up and divided by eight, which results in a total score between 0 and 3. The HAQ-DI is particularly, but not exclusively, used in rheumatic disease studies.

The FM-survey questionnaire was used to assess whether patients met the modified American College of Rheumatology 2010 classification criteria for FM. ${ }^{33}$ These criteria provide a severity scale for symptoms characteristic of FM, consisting of the widespread pain index (WPI) and symptomseverity (SS) score. The WPI consists of 19 possible painful body areas, and the SS score includes items about 6 symptoms. Patients satisfy the FM-survey criteria if the following conditions are met: WPI $\geq 7$ and SS $\geq 5$ or WPI 3-6 and SS $\geq 9$. The Dutch FM-survey questionnaire has not undergone formal psychometric testing, but both the WPI and SS are already frequently used in Dutch patient populations. ${ }^{34,35}$

Neuropathic-like pain features were assessed with the PDQ, a screening tool that was initially validated in patients with low-back pain. ${ }^{28,36}$ This questionnaire consists of seven questions evaluating pain qualities, one item about the course pattern of pain, and one item about pain radiation. An overall score of $1-38$ is generated. A score $\geq 19$ indicates likely neuropathic pain, 13-18 indicates possible neuropathic pain, and $\leq 12$ unlikely neuropathic pain. Additionally, the PDQ contains three 0-10 numeric rating scales for current, strongest, and average pain severity.

\section{Statistical analysis}

Descriptive statistics are presented as mean \pm SD when continuous and normally distributed and medians with IQR when continuous and non-normally distributed. Categorical variables are presented as numbers with percentages. Differences between patients with FM and RA were tested using independent $t$-tests or Mann-Whitney $U$ tests for non-normally distributed variables and $\chi^{2}$ tests for categorical variables. $P<0.05$ (two-tailed) was chosen to define statistical significance. Analyses were performed with IBM SPSS version 23.

Scaling properties of the seven preliminary items were examined using the models of monotone and double homogeneity $^{37}$ with the Mokken package in $\mathrm{R}$. The model of monotone homogeneity applies if all items measure the same latent variable and the expected item scores are monotonically non-decreasing throughout the observed score continuum. The first assumption was evaluated using the automatic itemselection procedure with the item-selection algorithm using different lower-bound values for the Loevinger coefficient of scalability $\left(H_{\mathrm{i}}\right)$, as suggested by Hemker et al. ${ }^{38}$ The second assumption was evaluated using the check.monotonicity function. The minimum size of rest-score groups was set at $n=30$.

The more restrictive model of double homogeneity applies if in addition to the assumptions already tested, the same ordering of items by their expected scores applies to all patients, irrespective of their total score. This is a desirable property for a scale, because it supports inferring from a patient's total score which specific symptom intensities are 
likely to be present. We used the backward-item-elimination procedure for polytomous responses proposed by Ligtvoet et al. ${ }^{39}$ The minimum size of rest-score groups was again set at $n=30$. Measurement properties of the resulting scale were examined. First, we obtained $H_{\mathrm{i}}$ coefficients for each item. If all items have high $H_{\mathrm{i}}$ values, this suggests that they discriminate well between people with different symptom-intensity levels and/or that items are well spread out across the latent variable. When aggregated over all items in a scale, values $>0.5$ are considered to indicate a strong scale. The overall accuracy of invariant item ordering was examined using the $H^{\mathrm{T}}$ coefficient. Finally, the Molenaar-Sijtsma method was used to estimate reliability of the total score. Coefficients $>0.70$ and $>0.90$ are generally taken to be sufficient for group- and individual-level use, respectively. ${ }^{37}$ Items that satisfied the criteria of Mokken-scale analysis were included in the final GPQ.

Next, we investigated associations of total GPQ scores with scores on the FM-survey questionnaire, PDQ, numeric rating-scale pain severity, SF36, and HAQ-DI to examine external construct validity. Associations were analyzed using Spearman correlations. Since FM is a condition characterized by widespread pain and hyperalgesia, we hypothesized that GPQ scores should correlate strongly $(r \geq 0.7)$ with scores on the FM-survey questionnaire. ${ }^{12,18-23,25,40}$ We also hypothesized that GPQ scores should correlate strongly with PDQ scores, because patients with generalized pain hypersensitivity and patients with neuropathic pain report very similar pain qualities (eg, allodynia and hyperalgesia). ${ }^{41-43}$ Furthermore, we hypothesized that GPQ scores should correlate strongly with pain-intensity scores. ${ }^{11,12,44}$ We also expected that GPQ scores should correlate moderately ( $r \geq 0.50)$ with health-related quality of life and more strongly with physical component scores than with mental component scores of the SF36, because we specifically referred to pain in the GPQ items. Finally, we hypothesized that GPQ scores should correlate at least moderately with scores on the HAQ-DI as well.

To examine the discriminative ability of GPQ scores, we compared total scores between patients with FM, which is generally considered a central sensitization-driven pain syndrome, ${ }^{12,17-23}$ and patients with RA, where pain is mainly attributed to peripheral inflammation of the joints. ${ }^{45}$ Additionally, receiver-operating characteristic (ROC) curve analyses were used to determine the accuracy and optimal cutoff value for the GPQ score for classifying patients with FM (vs RA). The area under the ROC curve (AUC) was used as an overall measure of classification accuracy of the GPQ. As a general rule of thumb, an AUC of $0.7-0.8$ is considered to indicate adequate discrimination and between 0.8 and 0.9 excellent discrimination. ${ }^{46}$ Optimal cutoff values were determined using Youden's index, which identifies the score with the highest combination of sensitivity and specificity. ${ }^{47}$

Using this cutoff value, the incremental validity of the GPQ in predicting FM above and beyond female sex, age, and pain severity as known predictors of FM was determined using a series of univariate and multivariate logistic regression analyses with associated ORs with 95\% CIs and Wald statistics. Pain severity was dichotomized to allow better interpretation and comparison of ORs of the different independent variables. According to Tubach et al, ${ }^{48}$ pain becomes clinically significant when it is $\geq 4$ out of 10 . Multivariable models were tested for goodness of fit with the HosmerLemeshow test, where small $P$-values indicate a lack of fit of the model. The overall goodness of fit was evaluated using Nagelkerke's pseudo- $R^{2}$.

\section{Results}

\section{Patient characteristics}

Although 99 FM patients returned the questionnaire (Table 1), 1 FM patient was excluded for not completing the GPQ, resulting in 98 evaluable FM patients. A total of 114 RA patients were included. Patients with FM were significantly more often women and were also $\sim 15$ years younger than RA patients on average. FM and RA patients did not differ significantly in other sociodemographic factors. Patients with FM reported more intense pain and used painkillers (both for conventional and for neuropathic pain) more often. Finally, FM patients reported worse health status in terms of more physical disabilities and a lower physical and mental quality of life.

\section{Psychometric evaluation of the GPQ}

The item-selection algorithm consistently yielded a single scale in which all seven items were included when lowerbound values for the $H_{\mathrm{i}}$ coefficient of up to $<0.6$ were used, supporting the unidimensionality of the GPQ items. In several iterations with a higher lower bound, either item 1 (light touch) or item 2 (rubbing skin) was omitted from the scales, but no secondary dimensions emerged. Although some violations of monotonicity were observed, none was statistically significant (Table 2). The results of these analyses support using the summed score for ordering patients along a single continuum of generalized pain hypersensitivity. No items showed violations of invariant item ordering, and thus no items were eliminated by the backward-elimination 
Table I Patient characteristics

\begin{tabular}{|c|c|c|c|}
\hline & FM $(n=98)$ & $\operatorname{RA}(n=1 \mid 4)$ & $P$-value \\
\hline Female, $\mathbf{n}(\%)$ & $88 / 98(89.8)$ & $76 / 114(66.7)$ & $<0.001$ \\
\hline Age (years), mean (SD) & $45.1(11.6)$ & $60.1(12.1)$ & $<0.001$ \\
\hline Disease duration (years), median (IQR) & $9.0(3.0-15.5)$ & $7.5(2.0-17.3)$ & 0.626 \\
\hline Education, n (\%) & & & 0.593 \\
\hline Low & $20 / 96(20.8)$ & $29 / 111(26.1)$ & \\
\hline Medium & $41 / 96(42.7)$ & $4 I / I I I(36.9)$ & \\
\hline High & $35 / 96(36.5)$ & $4 I / I I I(36.9)$ & \\
\hline Current smoking (yes), n (\%) & 23/97 (23.7) & $22 / 111(19.8)$ & 0.497 \\
\hline Current use of alcohol (yes), n (\%) & $54 / 98(55.1)$ & $72 / 111(64.9)$ & 0.150 \\
\hline \multicolumn{4}{|l|}{ Painkillers, self-reported current } \\
\hline Conventional (yes), n (\%) & $83 / 97(85.6)$ & $57 / 110(51.8)$ & $<0.001$ \\
\hline Neuropathic (yes), n (\%) & $18 / 96(18.8)$ & $0 / 110(0.0)$ & $<0.001$ \\
\hline \multicolumn{4}{|l|}{ Pain intensity (0-10), mean (SD) } \\
\hline Current pain & $6.7(1.7)$ & $3.1(2.6)$ & $<0.001$ \\
\hline Strongest pain, last 4 weeks & $8.1(1.3)$ & $4.5(3.0)$ & $<0.001$ \\
\hline Average pain, last 4 weeks & $6.9(1.5)$ & $3.6(2.6)$ & $<0.001$ \\
\hline PainDetect score, mean (SD) & $20.5(6.7)$ & $9.7(6.8)$ & $<0.001$ \\
\hline Unlikely, n (\%) & $17 / 95(17.9)$ & $70 / 109(64.2)$ & $<0.001$ \\
\hline Possible, n (\%) & I4/95 (I4.7) & $28 / 109(25.7)$ & $<0.001$ \\
\hline Likely, n (\%) & $64 / 95(67.4)$ & $11 / 109(10.1) 20 / 109$ & $<0.001$ \\
\hline FM-survey criteria positive, $n$ (\%) & $84 / 95(88.4)$ & $20 / 109(18.3)$ & $<0.001$ \\
\hline HAQ-DI (0-3), mean (SD) & $1.2(0.6)$ & $0.8(0.7)$ & $<0.001$ \\
\hline SF36 PCS (0-100), mean (SD) & $30.2(7.7)$ & $40.6(10.2)$ & $<0.001$ \\
\hline SF36 MCS (0-100), mean (SD) & $41.0(12.4)$ & $51.2(10.9)$ & $<0.001$ \\
\hline
\end{tabular}

Abbreviations: FM, fibromyalgia; HAQ-DI, Health Assessment Questionnaire - disability index; MCS, mental component summary; PCS, physical component summary; RA, rheumatoid arthritis; SF36, short-form health survey.

Table 2 Item scores and Mokken-scale analysis

\begin{tabular}{|c|c|c|c|c|c|c|c|c|}
\hline & \multirow[t]{2}{*}{ Mean (SD) } & \multirow[t]{2}{*}{$H_{\mathrm{i}}(\mathrm{SE})$} & \multicolumn{3}{|c|}{ Monotonicity } & \multicolumn{3}{|c|}{ Invariant item ordering } \\
\hline & & & Checks & Violations & $\begin{array}{l}\text { Significant } \\
\text { violations }\end{array}$ & Checks & Violations & $\begin{array}{l}\text { Significant } \\
\text { violations }\end{array}$ \\
\hline $\begin{array}{l}\text { I. Pain from light touch (eg, from a } \\
\text { pat on the back or handshake) }\end{array}$ & $1.53(1.15)$ & $0.64(0.04)$ & 37 & 0 & 0 & 27 & 0 & 0 \\
\hline $\begin{array}{l}\text { 2. Pain from friction on skin (eg, from } \\
\text { clothing or the wind) }\end{array}$ & $0.97(1.16)$ & $0.70(0.04)$ & 24 & 0 & 0 & 29 & 0 & 0 \\
\hline $\begin{array}{l}\text { 3. Pain from heat or cold that most } \\
\text { people would not experience as } \\
\text { painful (eg, from cold water or } \\
\text { holding cold objects) }\end{array}$ & $1.46(1.27)$ & $0.69(0.06)$ & 29 & 0 & 0 & 27 & 0 & 0 \\
\hline $\begin{array}{l}\text { 4. Pain that lasts longer than with } \\
\text { most other people }\end{array}$ & $2.02(1.35)$ & $0.65(0.07)$ & 41 & 0 & 0 & 28 & 0 & 0 \\
\hline $\begin{array}{l}\text { 5. Pain that arises only later and that } \\
\text { wouldn't arise in most other people } \\
\text { (eg, hours later or the next day after } \\
\text { exertion, such as walking) }\end{array}$ & $2.20(1.35)$ & $0.65(0.04)$ & 46 & 20 & 0 & 28 & 0 & 0 \\
\hline $\begin{array}{l}\text { 6. Unusually intense experiences of } \\
\text { pain (eg, nausea or gasping for air) }\end{array}$ & $1.14(1.42)$ & $0.64(0.06)$ & 30 & 0 & 0 & 27 & 0 & 0 \\
\hline $\begin{array}{l}\text { 7. Pain that also spreads to other } \\
\text { parts of the body (eg, pain in the } \\
\text { hand that spreads to the underarm } \\
\text { when holding objects) }\end{array}$ & $\mathrm{I} .8 \mathrm{I}(\mathrm{I} .37)$ & $0.64(0.04)$ & 44 & 2 & 0 & 28 & 0 & 0 \\
\hline
\end{tabular}


procedure. The overall conclusion of the Mokken analysis was that both the models of monotone and double homogeneity applied to the seven GPQ items.

$H_{\mathrm{i}}$ coefficients for individual items were all large, suggesting that each item usefully contributed to the ordering of patients on the latent metric (Table 2). The overall $H$ coefficient was 0.65 , which constitutes a strong scale $(>0.50)$, and $H^{\mathrm{T}}$ was 0.40 , which suggests medium accuracy of invariant item ordering according to Ligtvoet et al. ${ }^{39}$ Scores were highly reliable according to the SM coefficient $(r=0.90)$.

\section{Construct validity}

As expected, meeting FM-survey criteria, PDQ, painintensity, and HAQ-DI scores all correlated strongly $(\geq 0.7)$ with total GPQ scores (Table 3). In contrast to hypothesis, the GPQ correlated slightly more strongly with mental than with physical health-related quality of life.

\section{Discriminant validity}

As expected, patients with FM scored significantly higher on the GPQ compared to patients with RA. The mean total score for FM patients was 15.8 \pm 5.1 compared to $6.6 \pm 5.1$ for RA patients $(P<0.001)$. The ROC curve of the GPQ score for classifying patients with FM is presented in Figure 1. The GPQ had excellent accuracy in predicting FM, with an AUC of 0.89 (95\% CI 0.85-0.93). The optimal cutoff value for the GPQ in classifying FM was $>10$, with a sensitivity of $82.7 \%$, specificity of $77.2 \%$, positive predicted value of $75.7 \%$, negative predicted value of $83.8 \%$, and $79.7 \%$ correctly classified in total.

Univariate associations of generalized pain, pain intensity, female sex, and younger age with FM are presented in Table 4. A cutoff value $>10$ for the GPQ turned out to be one of the strongest predictors (OR 13.74) for FM. Female sex and

Table 3 Spearman correlations with GPQ total score

\begin{tabular}{|l|l|l|}
\hline & $\boldsymbol{r}$ & $\boldsymbol{P}$-value \\
\hline FM-survey criteria positive & 0.718 & $<0.00 \mathrm{I}$ \\
PainDetect total score & 0.870 & $<0.00 \mathrm{I}$ \\
Pain intensity & 0.805 & $<0.00 \mathrm{I}$ \\
Current pain (0-10) & 0.755 & $<0.00 \mathrm{I}$ \\
Strongest pain last 4 weeks (0-10) & 0.789 & $<0.00 \mathrm{I}$ \\
Average pain last 4 weeks (0-10) & 0.724 & $<0.00 \mathrm{I}$ \\
SF36 PCS (0-100) & -0.616 & $<0.001$ \\
SF36 MCS (0-100) & -0.749 & $<0.001$ \\
HAQ-DI (0-3) & 0.718 & $<0.001$ \\
\hline
\end{tabular}

Abbreviations: FM, fibromyalgia; GPQ, generalized pain questionnaire; HAQDI, Health assessment Questionnaire - disability index; MCS, mental component summary; PCS, physical component summary; SF36, Short Form health survey. age $<50$ years were both able independently to predict FM in a multivariate model (Table 4, step 1). Female sex was no longer an independent predictor of FM when the GPQ cutoff value of $>10$ was added to the model (step 2). A GPQ score $>10$ was the strongest predictor of FM in this model. Clinically relevant current pain severity was included last, since it was most strongly intercorrelated $(r=0.63)$ with a GPQ score $>10$, which could result in multicollinearity. Even after controlling for clinically relevant pain, a GPQ score $>10$ had strong incremental value in predicting FM. The final model had a good fit to the data, and together these four variables explained around $60 \%$ of the variance.

\section{Discussion}

In the present study, we introduced a short self-report instrument for assessing the presence and severity of various symptoms commonly associated with generalized pain hypersensitivity and provided an initial evaluation of its scaling properties and discriminative performance in patients with RA or FM. The findings showed that the seven-item GPQ has strong scaling properties and excellent accuracy in distinguishing between RA and FM pain phenotypes.

The results of psychometric evaluation using Mokkenscale analysis showed that a simple summed score can be used to measure patients' overall level of generalized pain hypersensitivity. Reliability of the scale was high $(r=0.90)$, suggesting that these summed scores can be used to monitor individuals or groups of patients over time and should be

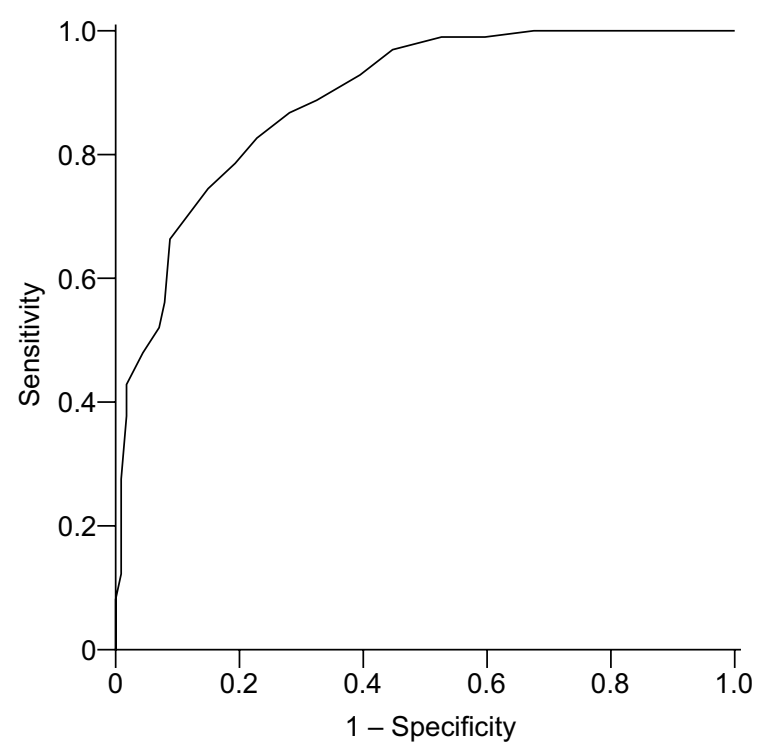

Figure I ROC curve of the GPQ for classifying patients with FM vs RA. Abbreviations: FM, fibromyalgia; GPQ, generalized pain questionnaire; RA, rheumatoid arthritis; ROC, receiver-operating characteristic. 
Table 4 Univariate and multivariate associations with FM

\begin{tabular}{|c|c|c|c|c|c|}
\hline Predictors & OR $(95 \% \mathrm{Cl})$ & $P$-value & Wald $(d f=I)$ test & HL $\chi^{2}$ test & Nagelkerke $R^{2}$ \\
\hline \multicolumn{6}{|c|}{ Univariate associations } \\
\hline GPQ total score $>10$ & $13.74(6.9 \mid-27.33)$ & $<0.001$ & 55.85 & & \\
\hline Female sex & $4.40(2.06-9.42)$ & $<0.001$ & 14.55 & & \\
\hline Age $<50$ years & $10.8 \mid(5.64-20.72)$ & $<0.001$ & 51.34 & & \\
\hline Clinically relevant pain & $19.65(7.81-49.47)$ & $<0.001$ & 40.00 & & \\
\hline \multicolumn{6}{|c|}{ Multivariate associations } \\
\hline Step I & & & & $4.622, P=0.099$ & 0.373 \\
\hline Female sex & $3.31(I .4 I-7.78)$ & 0.006 & 7.52 & & \\
\hline Age $<50$ years & $9.71(5.00-18.86)$ & $<0.001$ & 45.06 & & \\
\hline Step 2 & & & & $5.804, P=0.214$ & 0.584 \\
\hline Female sex & $2.80(0.91-8.64)$ & 0.073 & 3.21 & & \\
\hline Age $<50$ years & II $.02(4.76-25.53)$ & $<0.001$ & 31.37 & & \\
\hline GPQ total score $>10$ & $\mid 3.77(5.96-3|.8|)$ & $<0.001$ & 37.71 & & \\
\hline Step 3 & & & & $2.826, P=0.832$ & 0.606 \\
\hline Female sex & $2.70(0.84-8.64)$ & 0.095 & 2.78 & & \\
\hline Age $<50$ years & $10.59(4.34-25.85)$ & $<0.001$ & 26.91 & & \\
\hline GPQ total score $>10$ & $6.26(2.25-17.4 I)$ & $<0.001$ & 12.38 & & \\
\hline Clinically relevant pain & $4.73(1.36-16.54)$ & 0.015 & 5.93 & & \\
\hline
\end{tabular}

Abbreviations: FM, fibromyalgia; $\mathrm{HL}$, Hosmer-Lemeshow.

useful for discriminating between groups of patients with different levels of generalized pain hypersensitivity. ${ }^{49}$ Correlations with validated measures of similar concepts, such as FM-survey criteria and neuropathic-like pain features, were of the expected magnitude, supporting the validity of the scores.

This study additionally showed that the GPQ was useful for discriminating between patients with RA, characterized by localized pain in response to inflamed or damaged tissue, and patients with FM, characterized by widespread pain hyperresponsiveness. As expected, given these different pain phenotypes, FM patients reported a higher number and intensity of pain-hypersensitivity symptoms, and a GPQ score $>10$ was a predictor of FM that had strong incremental value in predicting FM beyond other known predictors, such as female sex, age, and clinically relevant pain severity. FM patients in the current study scored significantly worse than RA patients on other aspects of self-reported health measured for instance by the SF36 as well. However, as pain is an established determinant of such aspects of self-reported health, ${ }^{50}$ these were not included as covariates in the final multivariate model to avoid possible multicollinearity problems. Based on the current findings, a cutoff score $>10$ is suggested for identifying possible generalized pain hypersensitivity. However, more studies in different chronic pain populations are needed to confirm this value. Moreover, the current cutoff was determined based on the optimal combination of sensitivity and specificity only, so users specifically needing either high sensitivity or high specificity may also consider using a lower or higher cutoff score.

Despite recently increased interest in identifying the presence or assessing the severity of generalized pain hypersensitivity, the GPQ is the first dedicated self-report measure that specifically assesses commonly associated symptoms. Most studies to date have used short screeners originally developed for assessing neuropathic pain symptoms instead of assessing generalized pain hypersensitivity. For instance, several recent studies have suggested that the PDQ, a short neuropathic pain screener, may also be useful for identifying individuals with abnormal central pain processing. ${ }^{41,51-53}$ The GPQ arguably has higher content validity for assessing widespread pain hypersensitivity, since the questionnaire was developed based on typical symptoms for generalized pain hypersensitivity described in the literature, followed by a qualitative study with rheumatic patients with atypical pain symptoms suggestive of generalized pain hypersensitivity.

The Pain Sensitivity Questionnaire ${ }^{26}$ also measures selfreported pain sensitivity, but includes only items measuring intensity ratings of imagined painful situations, as opposed to actual complaints, such as those measured by the GPQ. Moreover, the Pain Sensitivity Questionnaire includes only items related to allodynia (eg, walking across a cool tiled floor with bare feet) and hyperalgesia (eg, trapping your finger in a drawer), and does not include other important signs of generalized pain hypersensitivity, such as aftersensations and spreading of pain. Finally, another frequently 
used instrument is the Central Sensitization Inventory. ${ }^{54}$ This assesses a long list of 25 health-related symptoms that are common to central sensitization syndromes in general. However, only five of these items focus on pain-related symptoms, whereas the other items assess a wide range of aspecific symptoms, such as difficulty concentrating and sensitivity to light. Consequently, its length and content also limit is usefulness for those interested in measuring generalized pain-hypersensitivity symptoms per se.

In the current study, FM patients completed the GPQ and other PROMs on paper, while RA patients completed these in a web-based questionnaire. In recent years, many studies have examined the equivalence of electronic and paper-andpencil administration of PROMs. In general, these studies show that PROMs administered on paper produce practically interchangeable scores compared with the same measures administered on an electronic device. ${ }^{55,56}$

No recall period is used for the items of the GPQ. Although this is quite common for similar questionnaires, such as those assessing neuropathic pain symtoms, ${ }^{28,57,58}$ there is considerable debate about the importance of using recall periods and selecting appropriate recall periods. For instance, the Patient-Reported Outcome Measurement Information System network explicitly advocates the use of recall periods that should be short enough to reduce recall errors and biases, but sufficiently long to capture a period of experience considered clinically relevant for outcome research. ${ }^{59}$ However, they also noted that there is currently little research available to inform the selection of an optimal recall period. ${ }^{59}$ The US Food and Drug Administration indicates that when using a recall period, the appropriateness of this period depends on the instrument's purpose and intended use. Also, the choice of a recall period should consider the variability, duration, frequency, and intensity of the concept measured, the disease or condition's characteristics, and the tested treatment. ${ }^{60}$ Because the GPQ was not intended to measure the exact frequency of symptoms in a certain period, but the severity of the presence of typical pain symptoms in general or in specific example situations (such as pain from cold water or holding cold objects), we preferred to avoid the use of any specific reference period. Additionally, there was no information available about how often these specific symptoms or situations typically occur in patients' daily lives that could be used to guide the selection of an appropriate recall period for all items. Although this is not expected to affect the discriminative ability of the GPQ negatively, it may reduce its sensitivity to change when used as an outcome measure. Future studies using the instrument in longitudinal observational or intervention studies are needed to examine this issue.
A limitation of this study is that we relied on a diagnosis of FM as a surrogate criterion for the presence of generalized pain hypersensitivity, since no true gold standard for diagnosing generalized pain hypersensitivity currently exists. Interestingly, only about $88 \%$ of the FM patients fulfilled the new American College of Rheumatology classification criteria for FM. ${ }^{33}$ One possible explanation is that in the current study, FM patients were identified using the ICD10 coding system. These patients were clinically diagnosed and not by means of the FM-survey criteria, which were developed for epidemiologic and clinical studies. For comparison, in the development study of the FM-survey criteria, only $60 \%$ of patients who had been previously (clinically) diagnosed with FM satisfied the criteria. ${ }^{33}$

Additionally, we used self-reported external instruments only to examine the construct validity of the scale. Future studies should further examine the criterion and construct validity of the GPQ by validating it against different methods of measuring pain, such as QST or imaging-based techniques, such as functional magnetic resonance imaging of the brain. Furthermore, the GPQ should be validated in other chronic pain populations, such as those in pain-rehabilitation settings. Finally, the results of the psychometric analysis of GPQ in the present study are encouraging for its use in the evaluation of the efficacy of therapies targeting generalized pain hypersensitivity. However, future studies are needed to confirm its responsiveness to change for such applications.

\section{Conclusion}

This study shows that the newly developed GPQ is psychometrically sound and has excellent accuracy in differentiating between chronic pain patients with pain presumably due to central nervous system hypersensitization (FM) and patients with pain primarily due to local nociception or inflammation (RA). Future validation studies are needed to examine its validity for identifying the presence of generalized pain hypersensitivity in other chronic pain populations and for monitoring changes in the extent of these pain symptoms over time or after treatment.

\section{Acknowledgment}

This work was supported by Lilly Netherlands, without restriction on publication.

\section{Author contributions}

MAHOV, PMTK, HEV and MAFJVDL conceptualized and designed the study. PFVB organized and supervised data collection and management. PFVB, MAHOV and PMTK analyzed the data. PFVB drafted the initial manuscript. All 
authors contributed toward data analysis, drafting and revising the paper, gave final approval of the version to be published and agree to be accountable for all aspects of the work.

\section{Disclosure}

The authors report no conflicts of interest in this work.

\section{References}

1. Blyth FM, March LM, Brnabic AJ, Jorm LR, Williamson M, Cousins MJ. Chronic pain in Australia: a prevalence study. Pain. 2001;89(2-3):127-134.

2. Breivik H, Collett B, Ventafridda V, Cohen R, Gallacher D. Survey of chronic pain in Europe: prevalence, impact on daily life, and treatment. Eur J Pain. 2006;10(4):287-333.

3. Johannes CB, Le TK, Zhou X, Johnston JA, Dworkin RH. The prevalence of chronic pain in United States adults: results of an Internet-based survey. J Pain. 2010;11(11):1230-1239.

4. Besson JM. Theneurobiology of pain. Lancet. 1999;353(9164):1610-1615.

5. Fornasari D. Pain mechanisms in patients with chronic pain. Clin Drug Investig. 2012;32(3699):45-52.

6. Woolf CJ, Salter MW. Neuronal plasticity: increasing the gain in pain. Science. 2000;288(5472):1765-1768.

7. Ji RR, Woolf CJ. Neuronal plasticity and signal transduction in nociceptive neurons: implications for the initiation and maintenance of pathological pain. Neurobiol Dis. 2001;8(1):1-10.

8. Ji RR, Kohno T, Moore KA, Woolf CJ. Central sensitization and LTP: do pain and memory share similar mechanisms? Trends Neurosci. 2003;26(12):696-705.

9. Schliessbach J, Siegenthaler A, Streitberger K, et al. The prevalence of widespread central hypersensitivity in chronic pain patients. Eur $J$ Pain. 2013;17(10):1-20.

10. Curatolo M, Uller M, Ashraf A. Pain hypersensitivity and spinal nociceptive hypersensitivity in chronic pain: prevalence and associated factors. Pain. 2015;156(11):1.

11. Latremoliere A, Woolf CJ. Central sensitization: a generator of pain hypersensitivity by central neural plasticity. J Pain. 2009;10(9):895-926.

12. Woolf CJ. Central sensitization: implications for the diagnosis and treatment of pain. Pain. 2011;152(3 Suppl):S2-S15.

13. International Association for the Study of Pain Taxonomy. Available from: https://www.iasp-pain.org/Education/Content.aspx?ItemNumber=1698. Accessed December 28, 2018.

14. Li J, Simone DA, Larson AA. Windup leads to characteristics of central sensitization. Pain. 1999;79(1):75-82.

15. Staud R. Evidence for shared pain mechanisms in osteoarthritis, low back pain, and fibromyalgia. Curr Rheumatol Rep. 2011;13(6):513-520.

16. Ren K, Dubner R. Descending modulation in persistent pain: an update. Pain. 2002;100(1-2):1-6.

17. Wolfe F, Rasker JJ. Fibromyalgia. In: Firestein GS, Budd RC, Gabriel E, McInnes IB, O'Dell JR, editors. Kelley's Textbook of Rheumatology. 9th ed. Philadelphia, PA: Saunders/Elsevier; 2013:733-751.

18. Vierck CJ. Mechanisms underlying development of spatially distributed chronic pain (fibromyalgia). Pain. 2006;124(3):242-263.

19. Petzke F, Clauw DJ, Ambrose K, Khine A, Gracely RH. Increased pain sensitivity in fibromyalgia: effects of stimulus type and mode of presentation. Pain. 2003;105(3):403-413.

20. McDermid AJ, Rollman GB, McCain GA. Generalized hypervigilance in fibromyalgia: evidence of perceptual amplification. Pain. 1996;66(2-3):133-144.

21. Staud R. Evidence of involvement of central neural mechanisms in generating fibromyalgia pain. Curr Rheumatol Rep. 2002;4(4): 299-305.

22. Staud R, Vierck CJ, Cannon RL, Mauderli AP, Price DD. Abnormal sensitization and temporal summation of second pain (wind-up) in patients with fibromyalgia syndrome. Pain. 2001;91(1-2):165-175.
23. Staud R, Robinson ME, Price DD. Temporal summation of second pain and its maintenance are useful for characterizing widespread central sensitization of fibromyalgia patients. J Pain. 2007;8(11):893-901.

24. Hurtig IM, Raak RI, Kendall SA, Gerdle B, Wahren LK. Quantitative sensory testing in fibromyalgia patients and in healthy subjects: identification of subgroups. Clin J Pain. 2001;17(4):316-322.

25. Desmeules JA, Cedraschi C, Rapiti E, et al. Neurophysiologic evidence for a central sensitization in patients with fibromyalgia. Arthritis Rheum. 2003;48(5):1420-1429.

26. Ruscheweyh R, Marziniak M, Stumpenhorst F, Reinholz J, Knecht S. Pain sensitivity can be assessed by self-rating: development and validation of the Pain Sensitivity Questionnaire. Pain. 2009;146(1-2):65-74.

27. Koop SM, Ten Klooster PM, Vonkeman HE, Steunebrink LM, van de Laar MA. Neuropathic-like pain features and cross-sectional associations in rheumatoid arthritis. Arthritis Res Ther. 2015;17(1):237.

28. Freynhagen R, Baron R, Gockel U, Tölle TR. painDETECT: a new screening questionnaire to identify neuropathic components in patients with back pain. Curr Med Res Opin. 2006;22(10):1911-1920.

29. Ware JE, Je WJ. SF36 health survey update. Spine. 2000;25(24): 3130-3139.

30. ten Klooster PM, Vonkeman HE, Taal E, et al. Performance of the Dutch SF36 version 2 as a measure of health-related quality of life in patients with rheumatoid arthritis. Health Qual Life Outcomes. 2013;11(1):77.

31. Fries JF, Spitz PW, Young DY. The dimensions of health outcomes: the health assessment questionnaire, disability and pain scales. JRheumatol. 1982;9(5):789-793.

32. ten Klooster PM, Taal E, van de Laar MA. Rasch analysis of the Dutch Health Assessment Questionnaire disability index and the Health Assessment Questionnaire II in patients with rheumatoid arthritis. Arthritis Rheum. 2008;59(12):1721-1728.

33. Wolfe F, Clauw DJ, Fitzcharles MA, et al. Fibromyalgia criteria and severity scales for clinical and epidemiological studies: a modification of the ACR preliminary diagnostic criteria for fibromyalgia. J Rheumatol. 2011;38(6):1113-1122.

34. Oudejans L, He X, Niesters M, Dahan A, Brines M, van Velzen M. Cornea nerve fiber quantification and construction of phenotypes in patients with fibromyalgia. Sci Rep. 2016;6(1):23573.

35. van Wilgen CP, Vuijk PJ, Kregel J, et al. Psychological distress and widespread pain contribute to the variance of the central sensitization inventory: a cross-sectional study in patients with chronic pain. Pain Pract. 2018;18(2):239-246.

36. Timmerman H, Wolff AP, Schreyer T, et al. Cross-cultural adaptation to the Dutch language of the PainDETECT-Questionnaire. Pain Pract. 2013;13(3):206-214.

37. van Schuur WH. Ordinal Item Response Theory: Mokken Scale Analysis. Thousand Oaks, CA: SAGE Publications; 2011.

38. Hemker BT, Sijtsma K, Molenaar IW. Selection of unidimensional scales from a multidimensional item bank in the polytomous Mokken I RT model. Appl Psychol Meas. 1995;19(4):337-352.

39. Ligtvoet R, van der Ark LA, Te Marvelde JM, Sijtsma K. Investigating an invariant item ordering for polytomously scored items. Educ Psychol Meas. 2010;70(4):578-595.

40. Staud R. Abnormal pain modulation in patients with spatially distributed chronic pain: fibromyalgia. Rheum Dis Clin North Am. 2009;35(2): 263-274.

41. Hochman JR, Davis AM, Elkayam J, Gagliese L, Hawker GA. Neuropathic pain symptoms on the modified PainDetect correlate with signs of central sensitization in knee osteoarthritis. Osteoarthritis Cartilage. 2013;21(9):1236-1242.

42. Rifbjerg-Madsen S, Christensen AW, Boesen M, et al. FRI0099 indications of reversibility of central sensitization according to the paindetect questionnaire in patients with rheumatoid arthritis: results from The Prospective Frame-Cohort Study. Ann Rheum Dis. 2016;75(Suppl 2):463.2-46464.

43. Ahmed S, Magan T, Vargas M, Harrison A, Sofat N. Use of the PainDetect tool in rheumatoid arthritis suggests neuropathic and sensitization components in pain reporting. J Pain Res. 2014;7:579-588. 
44. Meeus M, Nijs J. Central sensitization: a biopsychosocial explanation for chronic widespread pain in patients with fibromyalgia and chronic fatigue syndrome. Clin Rheumatol. 2007;26(4):465-473.

45. Lee YC. Effect and treatment of chronic pain in inflammatory arthritis. Curr Rheumatol Rep. 2013;15(1):300.

46. Hosmer DW, Lemeshow S. Applied Logistic Regression. 2nd ed. New York, NY: John Wiley \& Sons, Inc; 2000.

47. Youden WJ. Index for rating diagnostic tests. Cancer. 1950;3(1):32-35.

48. Tubach F, Ravaud P, Martin-Mola E, et al. Minimum clinically important improvement and patient acceptable symptom state in pain and function in rheumatoid arthritis, ankylosing spondylitis, chronic back pain, hand osteoarthritis, and hip and knee osteoarthritis: results from a prospective multina. Arthritis Care Res. 2012;64(11):1699-1707.

49. Aaronson N, Alonso J, Burnam A, et al. Assessing health status and quality-of-life instruments: attributes and review criteria. Qual Life Res. 2002;11(3):193-205.

50. Rupp I, Boshuizen HC, Dinant HJ, Jacobi CE, van den Bos GA. Disability and health-related quality of life among patients with rheumatoid arthritis: association with radiographic joint damage, disease activity, pain, and depressive symptoms. Scand J Rheumatol. 2006;35(3): 175-181.

51. Amris K, Jespersen A, Bliddal H. Self-reported somatosensory symptoms of neuropathic pain in fibromyalgia and chronic widespread pain correlate with tender point count and pressure-pain thresholds. Pain. 2010;151(3):664-669.

52. Moreton BJ, Tew V, das Nair R, Wheeler M, Walsh DA, Lincoln NB. Pain phenotype in people with knee osteoarthritis; classification and measurement properties of PainDetect and S-LANSS in a cross-sectional study. Arthritis Care Res. 2015;67(4):519-528.
53. Christensen AW, Rifbjerg-Madsen S, Christensen R, et al. Nonnociceptive pain in rheumatoid arthritis is frequent and affects disease activity estimation: cross-sectional data from the FRAME study. Scand J Rheumatol. 2016;45(6):461-469.

54. Mayer TG, Neblett R, Cohen H, et al. The development and psychometric validation of the central sensitization inventory. Pain Pract. 2012;12(4):276-285.

55. Gwaltney CJ, Shields AL, Shiffman S. Equivalence of electronic and paper-and-pencil administration of patient-reported outcome measures: a meta-analytic review. Value Health. 2008;11(2):322-333.

56. Muehlhausen W, Doll H, Quadri N, et al. Equivalence of electronic and paper administration of patient-reported outcome measures: a systematic review and meta-analysis of studies conducted between 2007 and 2013. Health Qual Life Outcomes. 2015;13(1):167.

57. Bennett MI, Smith BH, Torrance N, Potter J. The S-LANSS score for identifying pain of predominantly neuropathic origin: validation for use in clinical and postal research. J Pain. 2005;6(3):149-158.

58. Bouhassira D, Attal N, Alchaar H, et al. Comparison of pain syndromes associated with nervous or somatic lesions and development of a new neuropathic pain diagnostic questionnaire (DN4). Pain. 2005;114(1-2):29-36.

59. DeWalt DA, Rothrock N, Yount S, Stone AA; PROMIS Cooperative Group. Evaluation of item candidates: the PROMIS qualitative item review. Med Care. 2007;45(5 Suppl 1):S12-S21.

60. US Food and Drug Administration. Guidance for Industry: PatientReported Outcome Measures: Use in Medical Product Development to Support Labeling Claims; 2009. Available from: https://www.fda.gov/ downloads/drugs/guidances/ucm193282.pdf. Accessed December 28, 2018 .
Journal of Pain Research

\section{Publish your work in this journal}

The Journal of Pain Research is an international, peer reviewed, open access, online journal that welcomes laboratory and clinical findings in the fields of pain research and the prevention and management of pain. Original research, reviews, symposium reports, hypothesis formation and commentaries are all considered for publication.

\section{Dovepress}

The manuscript management system is completely online and includes a very quick and fair peer-review system, which is all easy to use. Visit http://www.dovepress.com/testimonials.php to read real quotes from published authors. 